\title{
Role of Additives to Overcome Limitations of Intermolecular Rhodium-Catalyzed Asymmetric Cyclopropanation
}

\author{
Jack C. Sharland, ${ }^{[a]}$ Bo Wei, ${ }^{[a]}$ David J. Hardee, ${ }^{[b]}$ Timothy R. Hodges, ${ }^{[b]}$ Wei Gong, ${ }^{[b]}$ Eric A. Voight, ${ }^{*[b]}$ \\ and Huw M. L. Davies ${ }^{*[a]}$
}
[a] Jack C. Sharland, Bo Wei, Huw M. L. Davies*
Department of Chemistry,
Emory University,
1515 Dickey Drive, Atlanta GA, 30322, United States
*hmdavie@emory.edu
[b] David J. Hardee, Timothy R. Hodges, Wei Gong, Eric A. Voight
Drug Discovery Science and Technology,
AbbVie,
1 North Waukegan Rd., North Chicago IL, 60064, United States
*eric.a.voight@abbvie.com

Supporting information for this article is given via a link at the end of the document.

\begin{abstract}
This study describes general methods for the enantioselective syntheses of disubstituted cyclopropane carboxylates including substitution patterns or heterocycle functionality previously observed as significant limitations. The key step is the dirhodium tetracarboxylate-catalyzed asymmetric cyclopropanation of vinyl arenes with aryl- or heteroaryldiazoacetates. The reactions are highly diastereoselective and high asymmetric induction could be achieved using either $(R)$-pantolactone as a chiral auxiliary or chiral dirhodium tetracarboxylate catalysts. For meta- or para-substituted aryl- or heteroaryldiazoacetates the optimum catalyst was $\mathrm{Rh}_{2}(R-p-\mathrm{Ph}-\mathrm{TPCP})_{4}$. In the case of ortho-substituted aryl- or heteroaryldiazoacetates, the optimum catalyst was $\mathrm{Rh}_{2}(R-$ TPPTTL)4. For a robust and generalizable reaction with the orthosubstituted substrates, 2-chloropyridine was required as an additive in the presence of either excess of $4 \AA$ molecular sieves or $1,1,1,3,3,3-$ hexafluoroisopropanol (HFIP). Under the optimized conditions, the cyclopropanation could be conducted in the presence of a variety of heterocycles, such as pyridines, pyrazines, quinolines, indoles, oxadiazoles, thiophenes and pyrazoles. The reaction can be readily conducted on a multigram scale and is compatible with aryldiazoacetate generated in situ without purification.
\end{abstract}

\section{Introduction}

The cyclopropane ring is a common structural motif incorporated into many pharmaceutical agents. ${ }^{[1]}$ Particularly common are 1,1 disubstituted cyclopropanes ${ }^{[2]}$ The two substituents are placed in a defined spacial arrangement to each other and the synthesis of such compounds is straightforward, as there is no additional chirality associated with the cyclopropane ring. In recent years more elaborate chiral cyclopropanes have been incorporated into therapeutic scaffolds, such as the trisubstituted cyclopropanes in beclabuvir (1), ${ }^{[3]}$ paritaprevir $(2)^{[4]}$ and glecaprevir $(3)^{[5]}$ (Figure 1 ). In these cases, three substituents are placed in a defined orientation. The syntheses of these cyclopropanes, however, are more challenging because they contain two stereogenic centers which need to be generated in a diastereoselective and enantioselective manner.

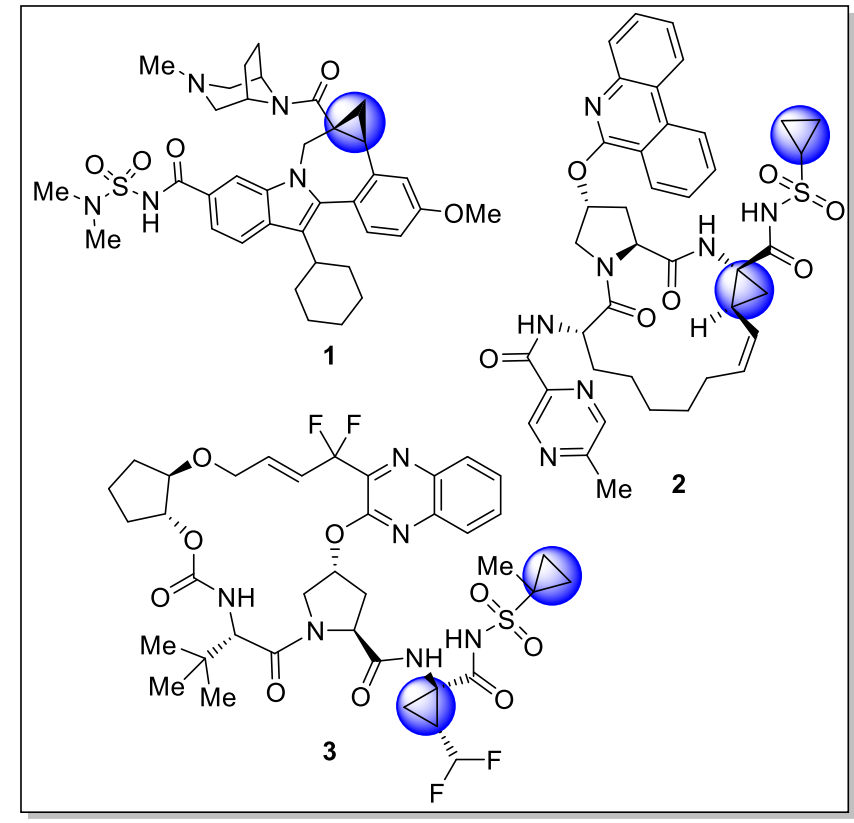

Figure 1. Examples of commercially available therapeutics containing highly substituted cyclopropanes. Beclabuvir (1), Paritaprevir (2), and Glecaprevir (3) therapeutics for the treatment of hepatitis-C (HCV).

A general method for the stereoselective synthesis of tri- or tetrasubstituted cyclopropanes is the rhodium-catalyzed cyclopropanation reactions of donor/acceptor carbenes. ${ }^{[6]} \mathrm{A}$ distinctive characteristic of this cyclopropanation is its high diastereoselectivity, typically $>30: 1$ d.r. ${ }^{[7]}$ Furthermore, effective methods are available to achieve asymmetric induction in the reaction by using either chiral auxiliaries ${ }^{[8]}$ or chiral catalysts. ${ }^{[7,9]}$ Having established the cyclopropanation chemistry, we became interested in developing a general method to synthesize cyclopropane carboxylates with heterocyclic functionality. The products could be chiral scaffolds of pharmaceutical interest that would be difficult to prepare by other means. The proposed method represents a significant challenge because the dirhodium catalysts and the rhodium-carbene intermediates are potentially susceptible to interactions with nucleophilic sites present in many heterocycles, which could interfere with the desired cyclopropanation unless carefully controlled. ${ }^{[10]}$

During our studies on cyclopropanation reactions with donor/acceptor carbenes, we developed two strategies for 
asymmetric induction. The first approach used $\alpha$-hydroxyesters as chiral auxiliaries, and $(R)$-pantolactone was found to be particularly effective. ${ }^{[8]}$ Soon thereafter, we developed chiral dirhodium tetracarboxylate catalysts for asymmetric cyclopropanation, ${ }^{[7]}$ The first generally effective catalysts were $\mathrm{N}$ sulfonylprolinate catalysts such as $\mathrm{Rh}_{2}(S-D O S P)_{4}(4){ }^{[7]}$ Since then, a variety of other chiral dirhodium catalysts have been developed. Three of these catalysts, $\mathrm{Rh}_{2}(R-\mathrm{PTAD})_{4},{ }^{\left[{ }^{9 a]}\right.} \mathrm{Rh}_{2}(R-p-$ $\mathrm{Ph}-\mathrm{TPCP}){ }_{4}{ }^{\left[{ }^{[\mathrm{c}]}\right]}$ and $\mathrm{Rh}_{2}(R-\mathrm{TPPTTL}) 4{ }^{[11]}(5,6$ and 7 respectively) play a significant role in the current study (Figure 2).

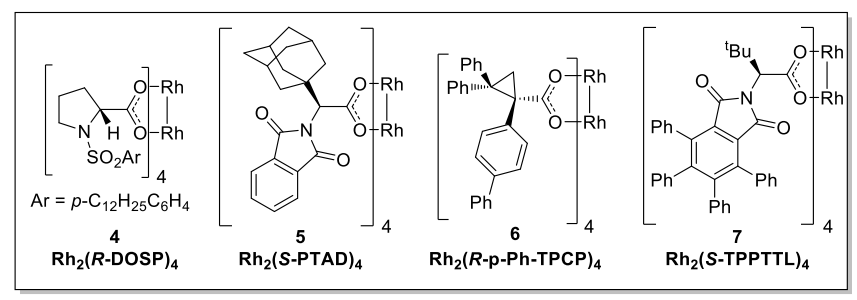

Figure 2. Structures of the key dirhodium catalysts used in this study.

Previous studies on cyclopropanation with heteroaryldiazoacetates as substrates gave mixed results.[9c, 12] The Rh2(S-DOSP)4-catalyzed cyclopropanation with methyl heteroaryldiazoacetates was generally high yielding and highly diastereoselective, but the levels of enantioselectivity were variable $(23-89 \%$ ee) (Scheme 1).[12b] It was evident that nucleophilic heterocycles such as pyridine tended to poison the catalyst and forcing conditions were often required for the cyclopropanation reaction to proceed. A 2-chloropyridyl functionality was better tolerated, presumably because the nitrogen is not as nucleophilic due to the inductive and steric effects of the 2-chloro substituent.[12b] More recently, a few trichloroethyl heteroaryldiazoacetates were shown to be capable of highly enantioselective cyclopropanation of styrene using Rh2(R-p-Ph-TPCP)4 as catalyst.[9c, 12a] Inspired by these positive results, we decided to conduct a systematic study to determine the scope of the heterocycles that can be incorporated in both the diazo compounds and in the trapping alkenes (Scheme 1). The study described herein consists of four stages. The first stage was conducted with chiral auxiliaries to gain rapid entry to the chiral cyclopropanes and avoid the potential interference of heterocyclic substrates with chiral catalysts. The second stage explored the use of chiral catalysts to achieve cyclopropanation of vinyl heterocycles with para- and meta-substituted aryl- and heteroaryldiazoacetates, which proceeded with high yield and selectivity according to established protocols. The third stage studied ortho-substituted diazo compounds, which required considerable optimization, leading to the discovery of additives with unexpected influence on the enantioselectivity. Finally, studies are described to scale-up the transformation for a multigram synthesis and protocols for generating and using the diazo compound in situ.

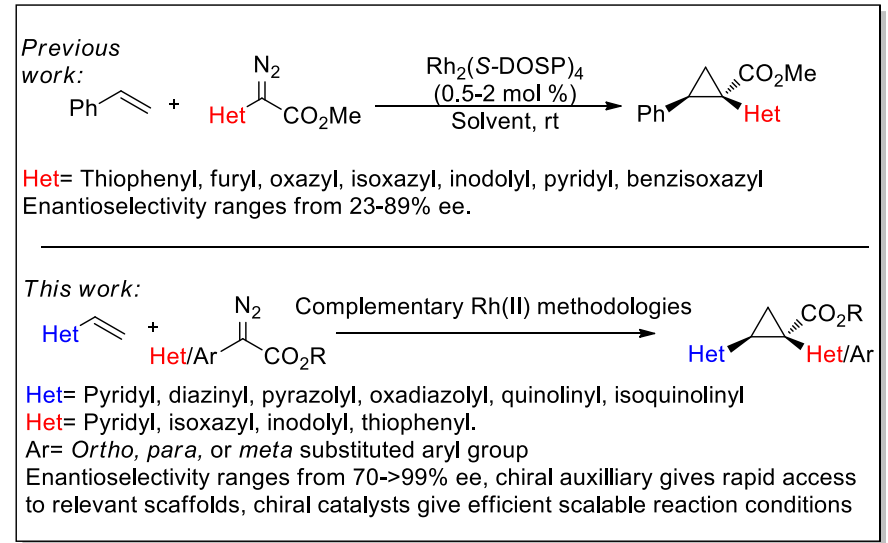

Scheme 1. Previous methodology used to prepare cyclopropyl heterocycles had limited success compared with the complementary strategy described herein.

\section{Results and Discussion}

At the outset of this project we required rapid access to chiral 1,2diaryl(heteroaryl) cyclopropanecarboxylates. ${ }^{[5 b, 9]}$ We began by examining the chiral auxiliary approach using $(R)$-pantolactone..$^{[8]}$ This approach is applicable to a wide range of substrates as summarized in Scheme 2. When applied towards the cyclopropanation of various vinyl heterocycles, ${ }^{[13]}$ the $(R)$ pantolactone-condensed-aryldiazoacetates gave routinely high asymmetric induction (87-98\% de) and the process was suitable for the synthesis of a variety of heterocycle-substituted cyclopropanes. In general, reactions involving a para-substituted aryldiazoacetate gave slightly higher asymmetric induction than ortho-substituted analogues (8-11 (97-98\% de) vs 9-16 (87-89\% de)). The absolute stereochemistry of $\mathbf{8 - 1 6}$ is tentatively assigned by analogy to the previously determined $\mathrm{Si}$ face selectivity exhibited by $(R)$-pantolactone in the reactions of donor/acceptor carbenes. ${ }^{[8]}$ While the chiral auxiliary approach proved generally effective, it does have limitations. The use of a stoichiometric chiral auxiliary is undesirable on large-scale due the cost and additional synthetic steps incurred for its installation and eventual removal. Additionally, only one of the enantiomers of pantolactone is inexpensive, limiting the accessibility to one enantiomer of the cyclopropane product ${ }^{[14]}$ For these reasons, while the $(R)$-pantolactone approach was useful for synthesizing a number of compounds in a short period of time, more contemporary methods using chiral catalysts were desirable. 


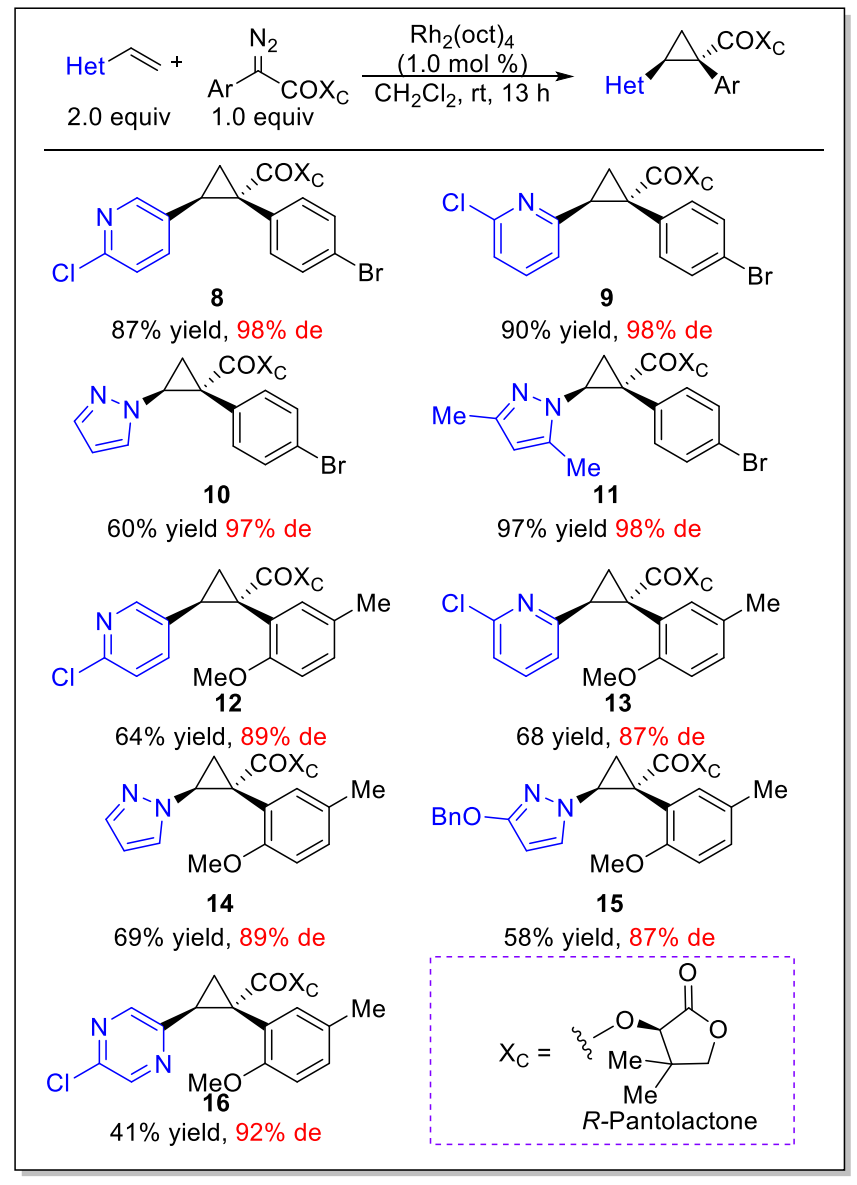

Scheme 2. Scope of heterocycle-containing cyclopropanes including the chiral auxiliary $(R)$-pantolactone. Reaction was run on $0.20 \mathrm{mmol}$ scale at room temperature using aryldiazoacetate (1.0 equiv), vinyl-heterocycle (2.0 equiv) and $1.0 \mathrm{~mol} \% \mathrm{Rh}_{2}(\mathrm{oct})_{4}(0.2 \mu \mathrm{mol})$ with $\mathrm{CH}_{2} \mathrm{Cl}_{2}$ as solvent.

There are few previously reported examples of highly enantioselective dirhodium-catalyzed cyclopropanation involving heteroaryldiazoacetates. ${ }^{[9 c, 12]}$ Even in the case of successful methodologies, the vast excess of substrate typically used in these reactions raises concerns that vinyl heterocycles, particularly pyridine derivatives, may interfere with the catalyst ${ }^{[10 a}$ ${ }^{15]}$ In order to evaluate the influence of different heterocycles an assortment of vinyl heterocycles ( 2.32 equiv, see SI for synthetic $\left.\operatorname{details}^{[13]}\right)$ were reacted with 2,2,2-trichloroethyl 2-(4bromophenyl)-2-diazoacetate (1.0 equiv) (Table 1$)$. The catalyst selected for this study was $\mathrm{Rh}_{2}(R-p-\mathrm{Ph}-\mathrm{TPCP})_{4}(0.5 \mathrm{~mol} \%)$, which has been shown to be the most effective chiral dirhodium tetracarboxylate catalyst for the cyclopropanation of styrenes. ${ }^{\left[{ }^{[c]}\right]}$ During the course of this previous study, considerable variability in the enantioselectivity was observed unless 10 weight equiv of $4 \AA$ molecular sieves was added to the reactions. ${ }^{\left[{ }^{9 c]}\right.}$ Under these conditions, the reaction proved to be robust, generating a series of cyclopropanes 17-25 with high enantioselectivity (83->99\% ee). Either $(\mathrm{MeO})_{2} \mathrm{CO}$, the optimal solvent identified in the earlier study, ${ }^{9 \mathrm{c}}$ or $\mathrm{CH}_{2} \mathrm{Cl}_{2}$, a generally effective solvent for donor/acceptor carbene transformations, could be used while maintaining high enantioselectivity. The reactions were competent with various pyridine (17-21) and quinoline derivatives (22 and 23), as well as five-membered heterocycles (24 and 25). The reactions of 2chloro-5-vinyl pyridine were then conducted with a range of paraand meta-substituted methyl and trichloroethyl aryldiazoacetates and a styryldiazoacetate to generate the cyclopropanes 26-30 Again, the reactions proceeded with high enantioselectivity (89$98 \%$ ee) except for the case of the 3,4-dimethoxy derivative, which generated the cyclopropane $\mathbf{3 0}$ in only $70 \%$ ee. The final series of reactions generated cyclopropanes $\mathbf{3 1 - 3 4}$ (83-95\% ee) with two heteroaryl rings. The absolute configuration of $\mathbf{1 7}$ was determined by $\mathrm{X}$-ray crystallography. The absolute configuration of 18-34 are tentatively assigned by analogy.

The ortho-substituted aryldiazoacetates were particularly desirable substrates in this study, but unfortunately, the $\mathrm{Rh}_{2}(R-p-$ $\mathrm{Ph}$-TPCP) ${ }_{4}$-catalyzed process, using the conditions described in Table 1, was not successful. The test cyclopropanation of 2chloro-5-vinyl pyridine (35) (2.5 equiv), with the ortho-substituted aryldiazoacetate 36 under $\mathrm{Rh}_{2}(R-p-\mathrm{Ph}-\mathrm{TPCP})_{4}$-catalyzed reaction conditions generated the product 37 in only $30 \%$ yield and $15 \%$ ee (Table 2, entry 1 ). While the stereoselective cyclopropanation of styrene with ortho-chlorophenyldiazoacetate has been reported in the presence of a second-generation dirhodium tetracarboxylate catalyst, $\mathrm{Rh}_{2}(\mathrm{~S}-\mathrm{PTAD})_{4}(\mathbf{5}),{ }^{\left[{ }^{[a]}\right.}$ this transformation required pentane as solvent to ensure high asymmetric induction, which is incompatible with several of the vinyl heterocycles because of solubility issues. ${ }^{[12 b]}$ The $\mathrm{Rh}_{2}(R-$ DOSP) 4 -catalyzed reaction of 35 with 36 generated the cyclopropane $\mathbf{3 7}$ in only $22 \%$ ee (Table 2 , entry 2 ). Similarly, the $\mathrm{Rh}_{2}(\mathrm{~S}-\mathrm{PTAD})_{4}$-catalyzed reaction gave low enantioselectivity (26\% ee) (Table 2 , entry 3 ).

Due to the poor performance of the established catalysts, a catalyst screen of several of the newer catalysts was conducted (see supplementary information for details). $\mathrm{Rh}_{2}(R \text {-TPPTTL) })_{4}$ a recently developed catalyst with a unique selectivity profile, $\left.{ }^{[11}, 16\right]$ emerged as the optimal catalyst for this system, giving 37 in $88 \%$ yield and $66 \%$ ee (Table 2). Interestingly, reactions of para or meta substituted aryldiazoacetates give the opposite absolute configuration to the products obtained from reactions with orthosubstituted aryldiazoacetates as determined by X-ray crystallography. This suggests that the different steric profile of ortho-substituted aryldiazoacetates substantially alters the approach of the olefin with respect to the rhodium carbene intermediate. Optimization of the $\mathrm{Rh}_{2}(R \text {-TPPTTL })_{4}$-catalyzed reaction by changing solvent (entries 4-6) or changing from the methyl ester to trichloroethyl ester (entry 7), did not improve the reaction. Lowering the reaction temperature to $0{ }^{\circ} \mathrm{C}$ increased the level of enantioselectivity to $80 \%$ ee (entry 8 ). The most dramatic effect, however, was to increase the amount of the 2-chloro-5vinylpyridine (35) to 5 equiv, which resulted in the formation of $\mathbf{3 7}$ in $95 \%$ yield and $98 \%$ ee (entry 9). ${ }^{[10 b,}{ }^{17]}$ Even though the optimization studies resulted in a considerable improvement in the effectiveness of the reaction, we were concerned that the reaction would not be amenable to scale up with the use of 10 weight equiv of molecular sieves to substrate. We have recently reported that hexafluoroisopropanol (HFIP) has beneficial effects on certain rhodium-catalyzed carbene reactions. ${ }^{[16]}$ Therefore, we 
Table 1. Scope of vinyl heterocycles aryl-diazoacetates and heteroaryl-diazoacetates compatible with previously established high-TON cyclopropanation methodology in the presence of $\mathrm{Rh}_{2}(R-p-\mathrm{Ph}-\mathrm{TPCP}) 4$.

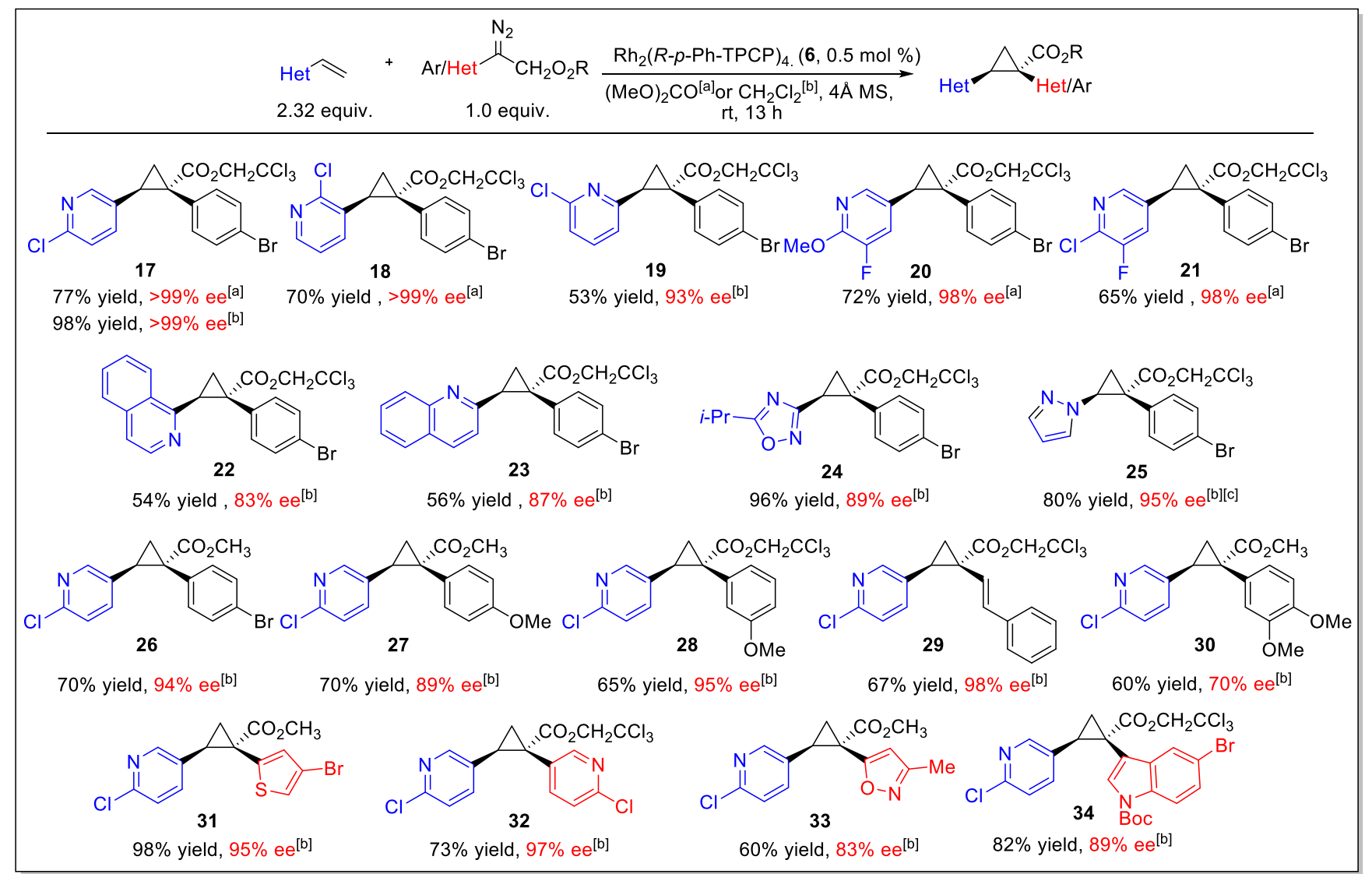

Reactions were conducted on $0.20 \mathrm{mmol}$ scale with 1.0 equiv of diazo-compound, 2.32 equivalents of vinyl-heterocycle, $0.5 \mathrm{~mol} \%$ catalyst loading ( $0.1 \mu \mathrm{mol})$ and either [a] $(\mathrm{MeO})_{2} \mathrm{CO}$ or [b] $\mathrm{CH}_{2} \mathrm{Cl}_{2}$ as solvent depending on solubility and optimal enantioselectivity obtained. \%Ee was determined by chiral $\mathrm{HPLC}$, absolute configuration of 17 was determined by X-ray crystallography (CCDC 2071127). [c] Reaction was conducted with 1.0 mol \% catalyst and run for $48 \mathrm{~h}$ at room temperature due to sluggish reactivity.

Table 2. Optimization of the enantioselective cyclopropanation of a vinyl-heterocycle with an ortho-substituted aryldiazoacetate.

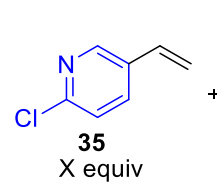<smiles>[R]Oc1ccc([N+]([O-])([O-])[O-])cc1C(=O)OC</smiles>

$\mathrm{Rh}_{2}(\mathrm{Cat})_{4}$ $\stackrel{(1 \mathrm{~mol} \%)}{\text { Solvent, Temp }}$ Additive, $13 \mathrm{~h}$<smiles>[R]OC1(c2ccc(C)cc2OC)C[C@@H]1c1ccc(Cl)nc1</smiles>

Entry Catalyst $\underset{{ }^{\circ} \mathrm{C}}{\mathrm{Tem}}$

Additive

Solvent

Equiv 35

$\mathrm{R}$

Yield, \% Ee, \%

\begin{tabular}{|c|c|}
\hline 1 & $\mathrm{Rh}_{2}(R-p-\mathrm{PhTPCP})_{4}(\mathbf{6})$ \\
\hline 2 & $\mathrm{Rh}_{2}(R \text {-DOSP })_{4}(\mathbf{4})$ \\
\hline 3 & $\mathrm{Rh}_{2}(\mathrm{~S}-\mathrm{PTAD})_{4}(\mathbf{5})$ \\
\hline 4 & $\mathrm{Rh}_{2}\left(R-\mathrm{TPPTTL}_{4}(\mathbf{7})\right.$ \\
\hline 5 & $\mathrm{Rh}_{2}(R \text {-TPPTTL })_{4}(\mathbf{7})$ \\
\hline 6 & $\mathrm{Rh}_{2}(R \text {-TPPTTL })_{4}(7)$ \\
\hline 7 & $\mathrm{Rh}_{2}(R \text {-TPPTTL })_{4}(\mathbf{7})$ \\
\hline 8 & $\mathrm{Rh}_{2}(R-\mathrm{TPPTTL})_{4}(\mathbf{7})$ \\
\hline 9 & $\mathrm{Rh}_{2}\left(R-\mathrm{TPPTTL}_{4}(7)\right.$ \\
\hline 10 & $\mathrm{Rh}_{2}(R \text {-TPPTTL })_{4}(7)$ \\
\hline
\end{tabular}

$4 \AA$ Mol sieves
$4 \AA$ Mol sieves
$4 \AA$ Mol sieves
$4 \AA$ Mol sieves
$4 \AA$ Mol sieves
$4 \AA$ Mol sieves
$4 \AA$ Mol sieves
$4 \AA$ Mol sieves
$4 \AA$ Mol sieves
HFIP

$\begin{array}{cr}\mathrm{CH}_{2} \mathrm{Cl}_{2} & 2.5 \\ \mathrm{CH}_{2} \mathrm{Cl}_{2} & 2.5 \\ \mathrm{CH}_{2} \mathrm{Cl}_{2} & 2.5 \\ \mathrm{CH}_{2} \mathrm{Cl}_{2} & 2.5 \\ (\mathrm{MeO})_{2} \mathrm{CO} & 2.5 \\ \mathrm{TFT} & 2.5 \\ \mathrm{TH}_{2} \mathrm{Cl}_{2} & 2.5 \\ \mathrm{CH}_{2} \mathrm{Cl}_{2} & 2.5 \\ \mathrm{CH}_{2} \mathrm{Cl}_{2} & 5.0 \\ \mathrm{CH}_{2} \mathrm{Cl}_{2} & 5.0\end{array}$

$\mathrm{CH}_{3}$
$\mathrm{CH}_{3}$
$\mathrm{CH}_{3}$
$\mathrm{CH}_{3}$
$\mathrm{CH}_{3}$
$\mathrm{CH}_{3}$
$\mathrm{CH}_{2} \mathrm{CCl}_{3}$
$\mathrm{CH}_{3}$
$\mathrm{CH}_{3}$
$\mathrm{CH}_{3}$

$\begin{array}{ll}30 & 15 \\ 68 & -22 \\ 70 & -26 \\ 88 & 66 \\ 78 & 43 \\ 58 & 58 \\ 47 & 39 \\ 85 & 80 \\ 95 & 98 \\ 93 & 92\end{array}$

All reactions were conducted on $0.20 \mathrm{mmol}$ scale using $1.0 \mathrm{~mol} \% \mathrm{Rh}_{2}(\mathrm{~S}-\mathrm{TPPTTL})_{4}(0.2 \mu \mathrm{mol})$ at room temperature.

decided to explore its effect on the optimized cyclopropanation, and we were pleased to observe that 10 equiv of HFIP could be used in place of the 10 weight equiv $4 \AA$ molecular sieves and retain high enantioinduction (entry 10). The optimized conditions developed in Table 2, were then applied to a range of substrates, but mixed results were obtained (Table 3). All of these reactions were conducted with the $(S)$ enantiomer of the catalyst. The $\mathrm{Rh}_{2}(\mathrm{~S} \text {-TPPTTL })_{4}$-catalyzed reactions of aryldiazoacetate $\mathbf{3 6}$ with various vinyl 2-chloropyridines to form the cyclopropanes 37-39 were highly enantioselective (90-98\% ee). In contrast the cyclopropanation of styrene with various 
ortho-substituted aryldiazoacetates generated the cyclopropanes 40-42 with low to moderate levels of enantioselectivity (4-64\% ee). Improved enantioselectivity was obtained in the formation of cyclopropane $\mathbf{4 3}$ (77\% ee), derived from a 2-chloropyridyldiazoacetate. The large variation in the levels of enantioselectivity was initially considered to be caused by trace impurities, but repeating the reactions with very carefully purified reagents or more dry conditions did not change the enantioselectivity. Finally, as 37-39 and 43 all contain a 2-chloropyridyl component and are formed with high levels of enantioselectivity, it was proposed that a 2chloropyridyl group may play a critical role in enhancing the enantioselectivity of the cyclopropanation. Such an effect would be consistent with the observed beneficial effect when using a large excess (5 equiv) of 2-chlorovinylpyridine seen in Table 2, entry 9. A control reaction was conducted to test this hypothesis. The cyclopropanation to form $\mathbf{4 0}$ was repeated in the presence of 1 equiv 2-chloropyridine as an additive. The modified conditions caused a dramatic effect on the enantioselectivity with $\mathbf{4 0}$ being formed in $\mathbf{9 5 \%}$ ee compared to $4 \%$ ee in the absence of the additive. A systematic study was conducted with a range of pyridine and quinoline analogs (see supplementary information for details), which revealed that 2choropyridine was the optimum additive. Pyridines lacking a substituent adjacent to nitrogen tended to poison the catalyst, but quinoline and other 2-substituted pyridines, such as 2methoxypyridine and 2-fluoropyridine, also provided considerable enhancement of enantioselectivity $(70-93 \%$ ee) but none proved superior to 2-chloropyridine in this reaction. The unexpected positive influence of 2-chloropyridine, prompted us to further evaluate its impact. The $\mathrm{Rh}_{2}(S-$ TPPTTL)4-catalyzed cyclopropanation of styrene with representative aryl- and pyridyldiazoacetates were examined and considerably different results were obtained depending whether or not the diazo compound had an ortho substituent. In the case of the cyclopropanes $\mathbf{4 1}$ and $\mathbf{4 2}$ derived from cyclopropanation of styrene with ortho-substituted aryldiazoacetates, the presence of 2-chloropyridine in the reaction improved the enantioselectivity from $55-77 \%$ ee to 84 $92 \%$ ee. In contrast to $\mathrm{Rh}_{2}(R-p-\mathrm{Ph}-\mathrm{TPCP})_{4}, \mathrm{Rh}_{2}(S-\mathrm{TPPTTL})_{4}$ is not an effective chiral catalyst for the formation of the cyclopropanes 44-46, derived from diazo compounds lacking ortho substituents. The enantioselectivity is low in the absence of additive $(29-48 \%$ ee) and even worse in the presence of 2chloropyridine $(0-41 \%$ ee). These studies demonstrated that while 2-chloropyridine as an additive can greatly enhance the enantioselectivity of $\mathrm{Rh}_{2}(S$-TPPTTL) 4 -catalyzed cyclopropanation, the effect is unique to ortho-substituted aryland heteroaryldiazoacetates.

Table 3. The substitution dependant effect of 2-chloropyridine on asymmetric cyclopropanation in the presence of $\mathrm{Rh}_{2}(\mathrm{~S}-\mathrm{TPPTTL})_{4}(7)$

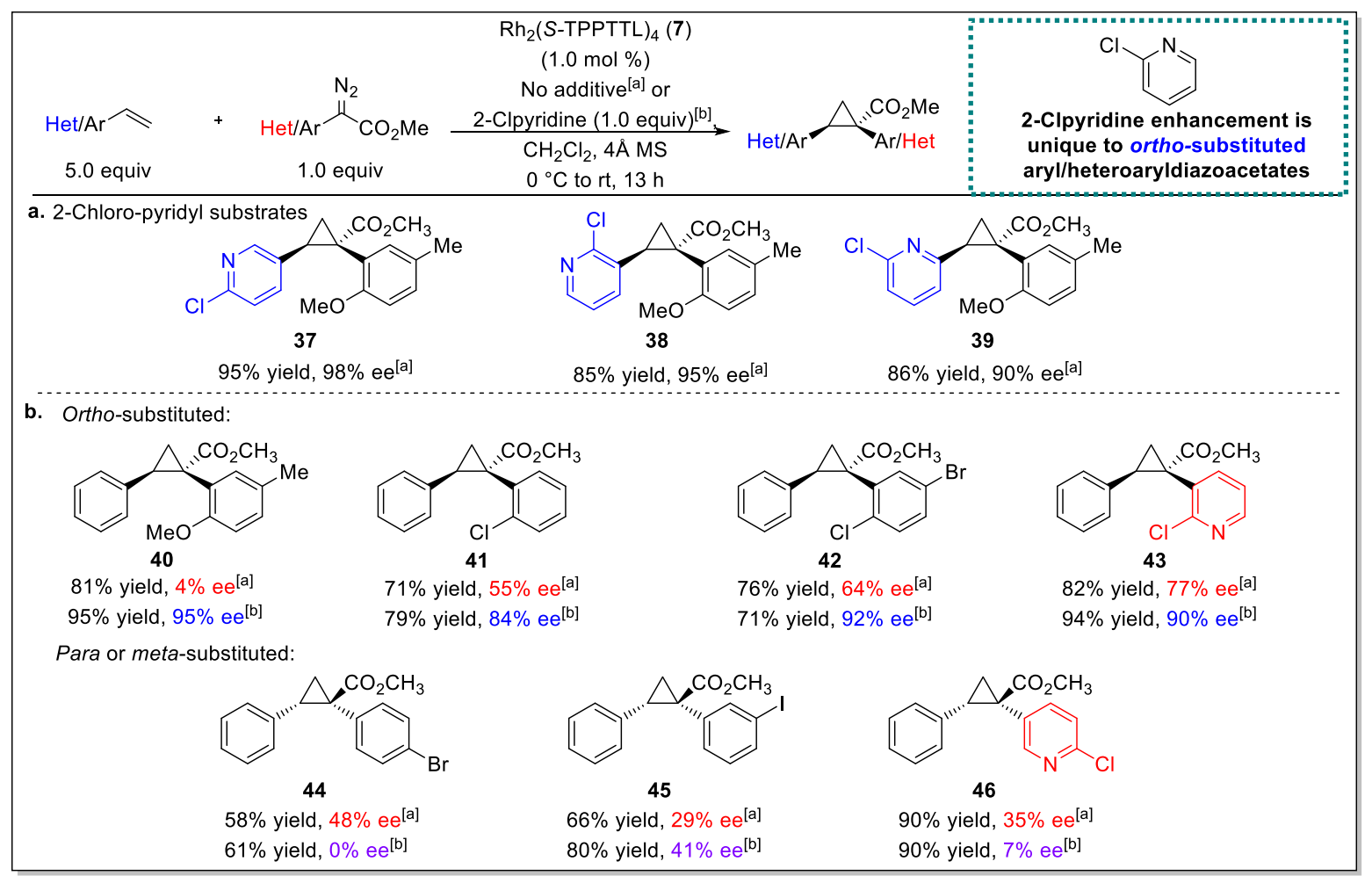

a. Initial scope of vinyl heterocycles compatible with ortho-aryldiazoacetate cyclopropanation. Reactions were conducted on $0.20 \mathrm{mmol}$ scale with 1.0 mol \% catalyst

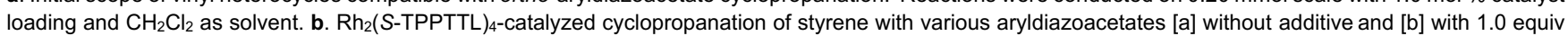
of 2-Clpyridine as a coordinating additive. Reactions were conducted on $0.20 \mathrm{mmol}$ scale with $1.0 \mathrm{~mol} \%$ catalyst loading $(0.2 \mu \mathrm{mol})$ and $\mathrm{CH}_{2} \mathrm{Cl}_{2}$ as solvent. The absolute configuration of $\mathbf{3 8}$ was determined by X-ray crystallography(CCDC 2071154). The absolute configuration of $\mathbf{3 7}$, $\mathbf{3 9 - 4 3}$ is tentatively assigned by analogy. 
Table 4. Scope of cyclopropanation with vinyl-heterocycles under the optimized ortho-aryldiazoacetate conditions.

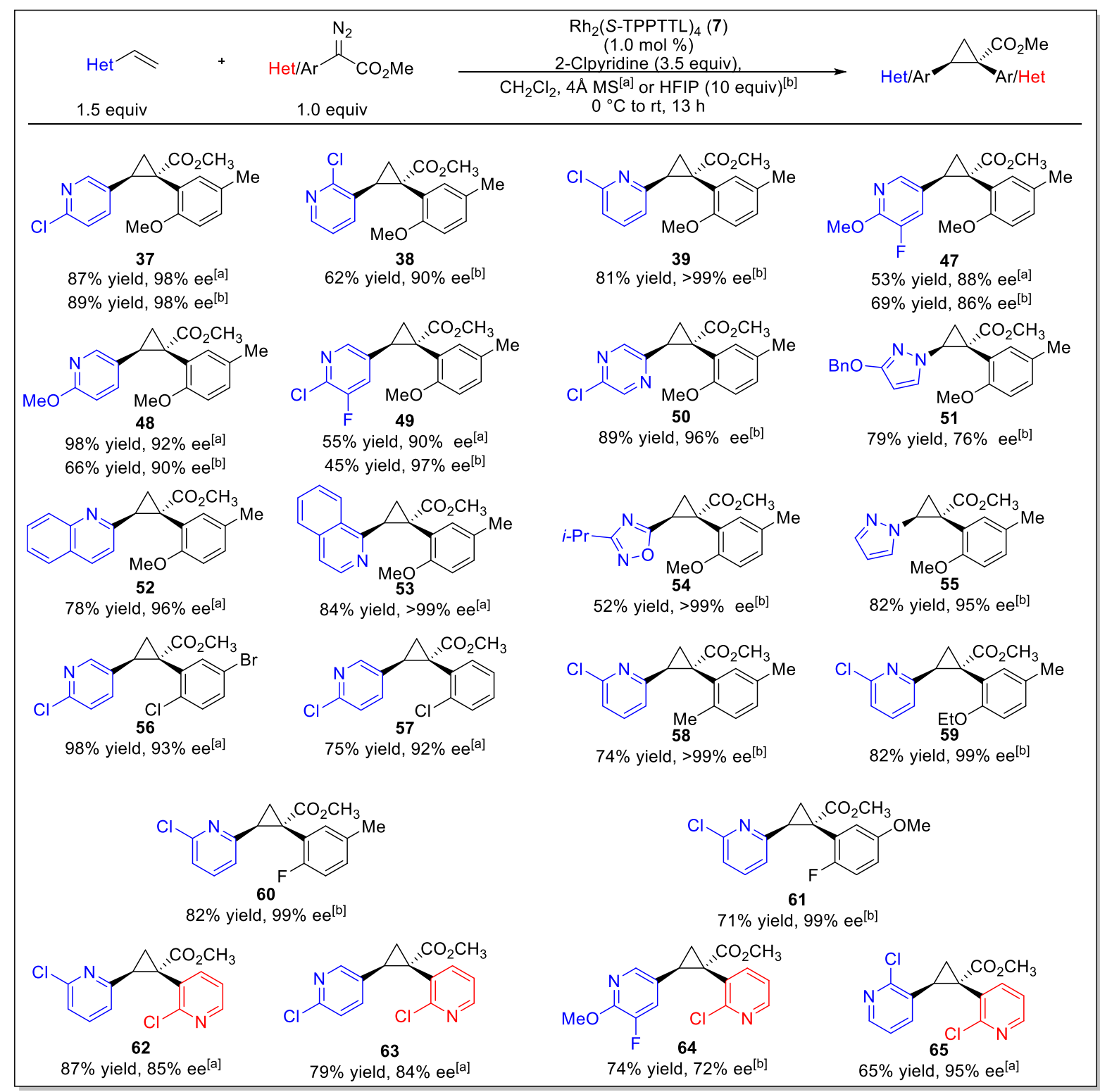

Reactions were conducted on $0.20 \mathrm{mmol}$ scale with $1.0 \mathrm{~mol} \% \mathrm{Rh}_{2}(\mathrm{~S} \text {-TPPTTL })_{4}(0.2 \mu \mathrm{mol}) \mathrm{CH}_{2} \mathrm{Cl}_{2}$ as solvent, and a reduced loading of vinyl-heterocycle (1.5 equiv) balanced out with 2-chloropyridine (3.5 equiv) with [a] 10 weight equiv. $4 \AA$ molecular sieves or [b] HFIP (10 equiv.). The absolute configuration of $37,39,47-65$ is tentatively assigned by analogy to that of 38 , which was determined by X-ray crystallography (CCDC 2071154).

Having established the positive influence of 2-chloropyridine in the cyclopropanation studies using styrene, the reactions of ortho- substituted aryldiazoacetates was examined with a range of vinyl heterocycles as illustrated in the formation of the cyclopropanes 47-55 (Table 4). As many of the vinyl heterocycles are expensive or are not commercially available the reactions were caried out with just 1.5 equiv of the vinyl heterocycle and 3.5 equiv of 2-chloropyridine. The reactions were compatible with a range of heterocycles, including pyridines, quinolines, isoquinolines, pyrazines, pyrazoles, and oxadiazoles. The reactions proceeded to form the cyclopropanes with generally very high enantioselectivity, ranging from $86 \%$ ee to $>99 \%$ ee. The reaction could also be conducted with methyl 2-(2-chloropyridin-3-yl)-2-diazoacetate and in this case, 1,2-diheteroarylcycloproane carboxylates 62 65 were formed in $72-95 \%$ ee. Effective reactions could be caried out using either $4 \AA$ molecular sieves or HFIP as coadditive. In the case of $\mathbf{3 7 , 4 7 - 4 9}$ the products were formed with high enantioselectivity using both sets of conditions. Exploratory studies were also conducted to determine whether the cyclopropanation reactions were amenable to scale-up. ${ }^{3,}$ ${ }^{18]}$ The replacement of molecular sieves with HFIP enabled the reaction to be performed on multi-gram scale, since the large quantity of sieves required to promote the reaction was considered prohibitive, providing 39 in $95 \%$ yield and $98 \%$ ee (Scheme 4). Performing the reaction on large scale also enabled the use of considerably lower catalyst loading $(0.16$ $\mathrm{mol} \%$ vs. $1.0 \mathrm{~mol} \%)$. The reaction was conducted at lower temperature to afford higher enantioselectivity, a common trend for dirhodium catalyzed asymmetric cyclopropanation by donor/acceptor carbenes. ${ }^{8}$ 


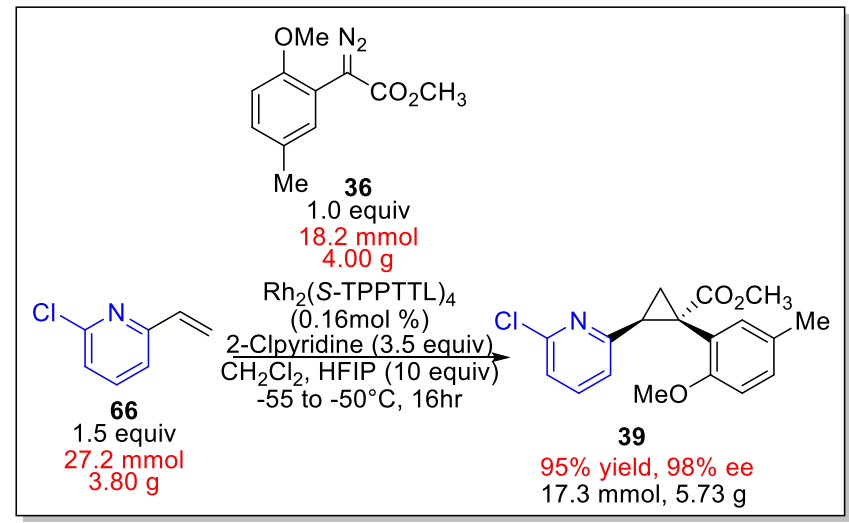

Scheme 4. Enantioselective cyclopropanation on a multi-gram scale.

Ultimately, for the reaction to be amenable to very large scale synthesis, the diazo compound would need to be generated in flow to avoid working with large quantities of a high energy intermediate. ${ }^{[19]}$ We have recently developed a copper-catalyzed method for the synthesis of diazo compounds from hydrazones, in which the only by-product is water. ${ }^{[20]}$ The copper catalyzedreaction is greatly accelerated with $N, N$ '-dimethylaminopyridine (DMAP), but DMAP, a very nucleophilic pyridine, would be expected to poison the catalyst or react with the carbene. Therefore, we have conducted exploratory studies to determine if the unpurified diazo compound from a copper-catalyzed oxidation can be directly used in the rhodium-catalyzed reaction. The copper-catalyzed oxidation of $\mathbf{6 7}$ in the presence of DMAP in air generated the desired diazo compound in essentially quantitative yield after stirring for $30 \mathrm{~min}$. Addition of the resulting solution to another reaction flask containing the reagents for a rhodiumcatalyzed cyclopropanation failed to proceed unless HFIP was present. In the presence of HFIP (20 equiv), the cyclopropane was formed in $83 \%$ yield and $98 \%$ ee (Scheme 5). The HFIP in this case is playing a very interesting role because it is deactivating the undesired effects of DMAP but still allowing the desirable influence of 2-chloropyridine to occur.

One of the most intriguing features of the current study is the dramatic role of additives on the reaction with ortho-substituted aryl- and heteroaryldiazoacetates. Typically, the enantioselectivity of rhodium-catalyzed cyclopropanation is not greatly influenced by trace moisture. Certainly, water will tend to cause a decrease in yield because it will competitively react with the carbene. In the case of ortho-substituted diazo compounds, trace moisture had a dramatically negative influence and a considerable excess ( 10 weight equiv) of $4 \AA$ molecular sieves was essential for reproducibly high enantioselectivity. Intriguingly, HFIP could be used in the place of molecular sieves and maintain similar levels of enantioselectivity. HFIP has been demonstrated to have a positive influence on a range of reactions, ${ }^{[21]}$ but the role of HFIP in rhodium-catalyzed cyclopropanation is not definitively known at this stage. Presumably, it is involved in hydrogen bonding and this blocks interference from the water. ${ }^{[21 b, 22]}$ The most unexpected effect was the role of 2-chloropyridine. In the absence of 2chloropyridine, the enantioselectivity of cyclopropanations involving ortho-substituted diazo compounds was very poor unless the substrate itself contained a 2-chloropyridyl functionality. In order to understand this better,

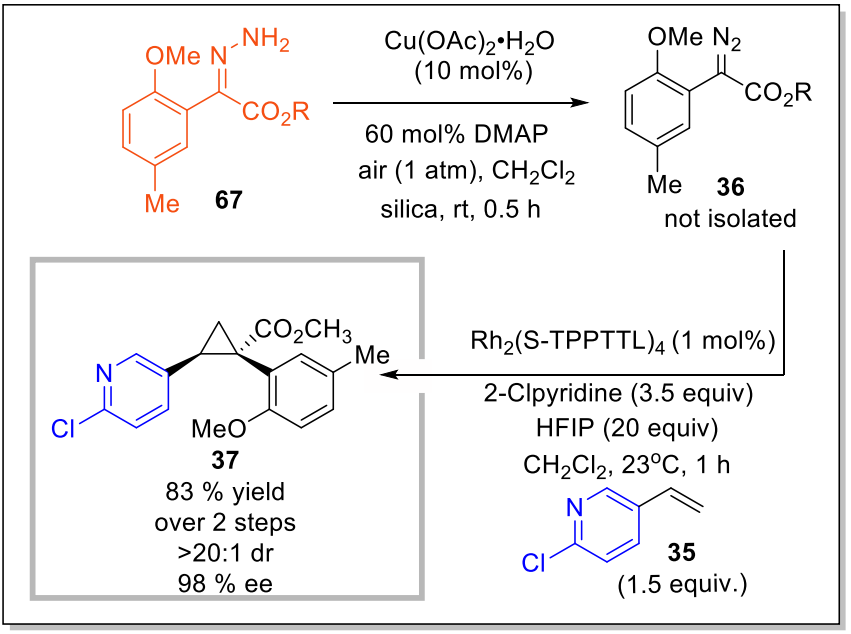

Scheme 5. Sequential copper-catalyzed diazo formation followed by a rhodiumcatalyzed cyclopropanation

crystals were grown of the 2-chloropyridine complex of $\mathrm{Rh}_{2}(\mathrm{~S}$ TPPTTL)4. The crystal structure, contained 2-chloropyridine molecules bound to each rhodium axial site and one additional 2chloropyridine situated within the bowl of the catalyst. An overlay of the previously reported crystal structure of the catalys ${ }^{[11]}$ and the 2-chloropyrdine-coordinated catalyst are shown in Figure 3. The 2-chloropyridine molecules have been removed for clarity (the full structure of the complex, is shown in the SI). An intriguing feature of the two overlaid structures is that one of the ligands has been considerably displaced upon coordination to the 2chloropyridine. This leads to an intriguing hypothesis that appropriate coordinating additives can alter the shape of the catalyst, which can then have a major influence on the asymmetric induction observed. Certainly, additives that would be expected to coordinate to the axial position of the dirhodium have been shown to influence the general outcome of carbene reactions, but the influence on enantioselectivity has not been extensively explored. ${ }^{[9 \mathrm{c}, 10 \mathrm{~b}, 17 \mathrm{e}, 23]}$ The cyclopropanation of $\mathbf{3 5}$ with 36 generated in situ illustrates the additive effects of HFIP and 2chloropyridine in concert. Without the presence of HFIP, the reaction cannot proceed, suggesting that DMAP acts as a poison to the rhodium catalyst, coordinating to the axial position and preventing carbene formation. However in the presence of HFIP, the DMAP cannot coordinate, suggesting an interaction between DMAP and HFIP, possibly through hydrogen bonding. 2Chloropyridine, however, is considerably less basic than DMAP, ${ }^{[24]}$ and apparently does not interact with HFIP in the same manner. As a result, the poisonous influence of DMAP is selectively deactivated while the beneficial coordination of 2chloropyridine proceeds undisturbed. 


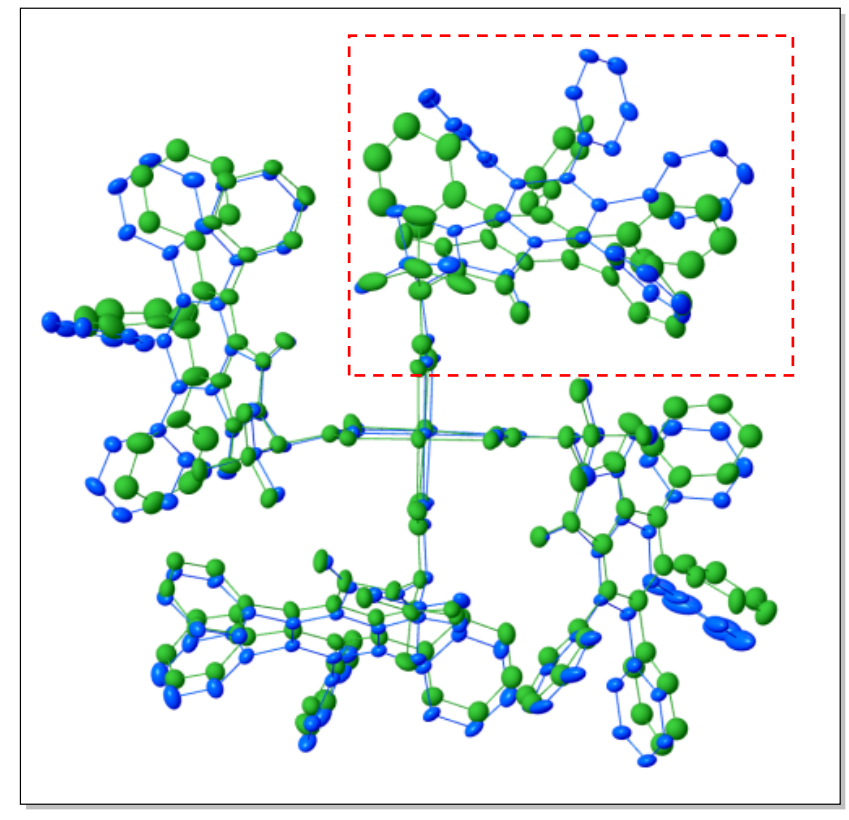

Figure 3. Structural perturbations in $\mathrm{Rh}_{2}(\mathrm{~S}-\mathrm{TPPTTL})_{4}$ enforced by the coordination of 2-chloropyridine based on X-ray analysis of a single crystal of 7 coordinated to 2-chloropyridine (CCDC 2071667). The top-right ligand is displaced from its original position (green) upon coordination with 2chloropyridine (blue). The axially coordinated ligands including 2-chlorpyridine ligand located inside of the bowl of the catalysts have been removed in order to give greater clarity of the overlaid structure of the catalysts

\section{Conclusion}

Complementary general methodologies for the syntheses of heterocycle-substituted cyclopropanes were developed. Use of $(R)$-pantolactone as a chiral auxiliary was identified as a fast and reliable way to synthesize a wide assortment of tri-substituted cyclopropanes stereoselectively. Alternatively, these compounds could be generated with high enantioselectivity using chiral catalysts. Para or meta-substituted aryldiazoacetates performed predictably and with high selectivity adapting recently reported cyclopropanation methodology using $\mathrm{Rh}_{2}(R-p-\mathrm{Ph}-\mathrm{TPCP})_{4}$ as catalyst. The reaction could be extended to several heteroaryldiazoacetates, enabling access to 1,2diheteroarylcyclopropane carboxylates. Ortho-substituted aryldiazoacetates, however, proved incompatible with these conditions and a different chiral catalyst, $\mathrm{Rh}_{2}(\mathrm{~S}$-TPPTTL) 4 was required. During these studies, the role of additives was found to have a major influence. 2-Chloropyridine was discovered as a coordinating additive capable of significantly enhancing the enantioselectivity of cyclopropanation involving ortho-substituted aryldiazoacetates. These efforts resulted in a robust and generalizable methodology which was performed on multi-gram scale and made more process-amenable by substituting $4 \AA$ molecular sieves for HFIP to desensitize the reaction to $\mathrm{H}_{2} \mathrm{O}$. This in situ desensitization was further exploited to perform the reaction with aryldiazoacetate generated in situ from the corresponding hydrazone using copper-catalyzed oxidation. These unique additive effects may have broad implications for other catalytic reaction manifolds.

\section{Experimental}

See SI for all experimental details. The following crystal structures have been deposited in the Cambridge Crystallographic Data Centre: 7 coordinated to 2-chloropyridine (CCDC 2071667), 17 (CCDC 2071127), and 38 (CCDC 2071154)

\section{Conflicts of interest}

The authors declare the following competing financial interests: H.M.L Davies is a named inventor on a patent entitled Dirhodium Catalyst Compositions and Synthetic Processes Related Thereto (US 8,974,428, issued 3/10/2015). D J. Hardee, T. R. Hodges, W. Gong, and E. A. Voight are employees of AbbVie. AbbVie contributed to the design, study conduct, and financial support for this research. AbbVie participated in the interpretation of data, review, and approval of the publication.

\section{Acknowledgements}

This work was supported by AbbVie. Additional support was provided by the National Science Foundation (CHE-1956154 and the $\mathrm{CCl}$ Center for Selective $\mathrm{C}-\mathrm{H}$ Functionalization (CHE1700982)). Instrumentation used in this work was supported by the National Science Foundation (CHE 1531620 and CHE 1626172). The authors would like to thank Dr. John Bacsa for his instrumental help in determining the crystal structure of the coordinated catalyst and absolute configuration of cyclopropanation products via X-Ray crystallography.

Keywords: Cyclopropanation $\cdot$ heterocycles $\cdot$ asymmetric catalysis $\cdot$ rhodium carbenoid $\cdot$ additive enhancement $\cdot$ HFIP

\section{References:}

[1] [a] A. Gagnon, M. Duplessis, L. Fader, Org. Prep. Proced. Int. 2010, 42, 1-69; [b] T. T. Talele, J. Med. Chem. 2016, 59, 8712-8756; [c] R. D. Taylor, M. MacCoss, A. D. G. Lawson, J. Med. Chem. 2014, 57, 5845-5859; [d] J. Salaiin, M. Bairtr, J Curr. Med. Chem 1995, 2, 511-542. [2] For examples of medicinally relevant molecules bearing 1,1-disubstituted cyclopropanes see: [a] M. A. Sala, M. Jain, Expert . Opin. Respir. Med. 2018, 12, 725-732; [b] T. W. Loo, M. C. Bartlett, D. M. Clarke, Biochem. Pharmacol. 2013, 86, 612-619; [c] Y. Jiang, S. W. Andrews, K. R. Condroski, B. Buckman, V. Serebryany, S. Wenglowsky, A. L. Kennedy, M. R. Madduru, B. Wang, M. Lyon, J. Med. Chem. 2014, 57, 1753-1769; [d] G. Shen, F. Zheng, D. Ren, F. Du, Q. Dong, Z. Wang, F. Zhao, R. Ahmad, J. Zhao, Am. J. Hematol. 2018, 11, 1-11; [e] J. O. Link, J. G. Taylor, L. Xu, M. Mitchell, H. Guo, H. Liu, D. Kato, T. Kirschberg, J. Sun, N. Squires, J. Med. Chem. 2014, 57, 2033-2046; [f] C. Köllmann, S. M. Wiechert, P. G. Jones, T. Pietschmann, D. B. Werz, Org. Lett. 2019, 21, 69666971.

[3] J. Bien, A. Davulcu, A. J. DelMonte, K. J. Fraunhoffer, Z. Gao, C. Hang, Y. Hsiao, W. Hu, K. Katipally, A. Littke, A. Pedro, Y. Qiu, M. Sandoval, R. Schild, M. Soltani, A. Tedesco, D. Vanyo, P. Vemishetti, R. E. Waltermire, Org. Process. Res. Dev. 2018, 22, 1393-1408.

[4] [a] A. F. Carrion, J. Gutierrez, P. Martin, Expert Opin. Pharmacother. 2014, 15, 711-716; [b] Caspi, D.D., Cink, R.D., Clyne, D., Diwan, M., Engstrom, K.M., Grieme, T., Mei, J., Miller, R.W., Mitchell, C., Napolitano, J.G. and Nere, N., Tetrahedron, 2019, 75, 4271-4286. 
[a] Y. N. Lamb, Drugs 2017, 77, 1797-1804. [b] Kallemeyn, J.M., Engstrom, K.M., Pelc, M.J., Lukin, K.A., Morrill, W.H., Wei, H., Towne, T.B., Henle, J., Nere, N.K., Welch, D.S. and Shekhar, S., Org. Process. Res. Dev, 2020, 24, 1373-1392. [c] Hill, D.R., Abrahamson, M.J., Lukin, K.A., Towne, T.B., Engstrom, K.M., Reddy, R.E., Kielbus, A.B., Pelc, M.J., Mei, J., Nere, N.K. and Chen, S., Org. Process. Res. Dev, 2020, 24,1393-1404.

[6] H. M. Davies, E. G. Antoulinakis, Org. React. 2004, 57, 1326.

[7] H. M. L. Davies, P. R. Bruzinski, D. H. Lake, N. Kong, M. J. Fall, J. Am. Chem. Soc. 1996, 118, 6897-6907.

[8] H. M. L. Davies, N. J. S. Huby, W. R. Cantrell, J. L. Olive, J. Am. Chem. Soc. 1993, 115, 9468-9479.

[9] [a] K. M. Chepiga, C. Qin, J. S. Alford, S. Chennamadhavuni, T. M. Gregg, J. P. Olson, H. M. L. Davies, Tetrahedron 2013, 69, 5765-5771; [b] S. Negretti, C. M. Cohen, J. J. Chang, D. M. Guptill, H. M. L. Davies, Tetrahedron 2015, 71, 7415-7420; [c] B. Wei, J. C. Sharland, P. Lin, S. M. Wilkerson-Hill, F. A. Fullilove, S. McKinnon, D. G. Blackmond, H. M. L. Davies, ACS Catal. 2020, 10, 1161-1170; [d] Z. Yu, A. Mendoza, ACS Catal. 2019, 9, 7870-7875. [e] Singha, S., Buchsteiner, M., Bistoni, G., Goddard, R., \& Fürstner, A., J. Am. Chem. Soc. 2021,

[10] [a] Q.-S. Ye, X.-N. Li, Y. Jin, J. Yu, Q.-W. Chang, J. Jiang, C.-X. Yan, J. Li, W.-P. Liu, Inorg. Chim. Acta. 2015, 434, 113-120; [b] T. D. Nelson, Z. J. Song, A. S. Thompson, M. Zhao, A. DeMarco, R. A. Reamer, M. F. Huntington, E. J. J. Grabowski, P. J. Reider, Tetrahedron. Lett. 2000, 41, 1877-1881.

[11] J. Fu, Z. Ren, J. Bacsa, D. G. Musaev, H. M. L. Davies, Nature 2018, 564, 395-399.

[12] [a] L. Fu, J. D. Mighion, E. A. Voight, H. M. Davies, Chem. Eur. J. 2017, 23, 3272-3275; [b] H. M. L. Davies, R. J. Townsend, J. Org. Chem. 2001, 66, 6595-6603.

[13] For earlier reports detailing the general methods that were used to synthesize vinyl-heterocycles see: [a] S. Y. Ko, J. Lerpiniere, I. D. Linney, R. Wrigglesworth, J. Chem. Soc. Chem. Commun. 1994, 1775-1776; [b] G. Adjabeng, T. Brenstrum, J. Wilson, C. Frampton, A. Robertson, J. Hillhouse, J. McNulty, A. Capretta, Org. Lett. 2003, 5, 953955.

[14] M. Calmes, J. Daunis, R. Jacquier, F. Natt, Org. Prep. Proced. Int. 1995, 27, 107-108.

[15] [a] A. Dennis, J. Korp, I. Bernal, R. Howard, J. Bear, Inorg. Chem. 1983, 22, 1522-1529; [b] M. Fandzloch, A. Augustyniak, L. Dobrzańska, T. Jędrzejewski, J. Sitkowski, M. Wypii, P. Golińska, J. Inorg. Biochem. 2020, 111072.

[16] J. Vaitla, Y. T. Boni, H. M. L. Davies, Angew. Chem. Int. Ed. 2020, 59, 7397-7402.

[17] For selected examples of additive enhanced rhodium carbenoid transformations see: [a] H. Lebel, H. Piras, J.
Bartholoméüs, Angew. Chem. Int. Ed. 2014, 53, 73007304; [b] M. Kim, J. Lee, H.-Y. Lee, S. Chang, Adv. Synth. Catal. 2009, 351, 1807-1812; [c] H. M. L. Davies, C. Venkataramani, Org. Lett. 2003, 5, 1403-1406; [d] B. G. Anderson, D. Cressy, J. J. Patel, C. F. Harris, G. P. A. Yap, J. F. Berry, A. Darko, Inorg. Chem. 2019, 58, 17281732; [e] A. F. Trindade, J. A. S. Coelho, C. A. M. Afonso, L. F. Veiros, P. M. P. Gois, ACS Catal. 2012, 2, 370-383. [a] J. H. Simpson, J. Godfrey, R. Fox, A. Kotnis, D. Kacsur, J. Hamm, M. Totelben, V. Rosso, R. Mueller, E. Delaney, Tetrahedron: Asymmetry 2003, 14, 3569-3574; [b] L. R. Marcin, D. J. Denhart, R. J. Mattson, Org. Lett. 2005, 7, 2651-2654; [c] R. Anthes, O. Bello, S. Benoit, C.K. Chen, E. Corbett, R. M. Corbett, A. J. DelMonte, S. Gingras, R. Livingston, J. Sausker, Development, Org. Process. Res. Dev. 2008, 12, 168-177; [d] J. R. Gage, F. Chen, C. Dong, M. A. Gonzalez, Y. Jiang, Y. Luo, M. D. McLaws, J. Tao, Development, Org. Process. Res. Dev. 2020, 24, 2025-2033.

[19] S. P. Green, K. M. Wheelhouse, A. D. Payne, J. P. Hallett, P. W. Miller, J. A. Bull, Org. Process. Res. Dev. 2020, 24, 67-84.

[20] L. W, J.Twilton, B. Wei, M. Lee, M. N. Hopkins, J. Bacsa, S. S. Stahl, H. M. L. Davies, J. Am. Chem. Soc. 2021, 11, 2676-2683

[21] For selected reports on reactions and properties of HFIP see: [a] S. Khaksar, J. Fluorine. Chem. 2015, 172, 51-61; [b] A. Berkessel, J. A. Adrio, D. Hüttenhain, J. M. Neudörfl, J. Am. Chem. Soc. 2006, 128, 8421-8426; [c] I. Colomer, A. E. Chamberlain, M. B. Haughey, T. J. Donohoe, Nat. Rev. Chem. 2017, 1, 1-12; [d] S. K. Sinha, T.

Bhattacharya, D. Maiti, React. Chem. Eng. 2019, 4, 244253; [e] V. Pozhydaiev, M. Power, V. Gandon, J. Moran, D. Lebœuf, Chem. Commun. 2020, 56, 11548-1156.; [f] Bhattacharya, T., Ghosh, A., \& Maiti, D. Chem. Sci. 2021, 12, 3857-3870; [g] Gray, E. E., Nielsen, M. K., Choquette, K. A., Kalow, J. A., Graham, T. J., \& Doyle, A. G. J. Am. Chem. Soc. 2016, 138, 10802-10805; [h] Jana, S., Yang, Z., Li, F., Empel, C., Ho, J., \& Koenigs, R. M., Angew. Chem. Int. Ed, 2020, 59, 5562-5566.

[22] [a] O. Hollóczki, A. Berkessel, J. Mars, M. Mezger, A. Wiebe, S. R. Waldvogel, B. Kirchner, ACS Catal. 2017, 7, 1846-1852; [b] M. Borrell, S. Gil-Caballero, M. Bietti, M. Costas, ACS Catal. 2020, 10, 4702-4709.

[23] [a] A. F. Trindade, P. M. Gois, L. F. Veiros, V. André, M. T. Duarte, C. A. Afonso, S. Caddick, F. G. N. Cloke, J. Org. Chem. 2008, 73, 4076-4086; [b] V. N. Lindsay, C. Nicolas, A. B. Charette, J. Am. Chem. Soc. 2011, 133, 8972-8981. N. A. Caballero, F. J. Melendez, C. Muñoz-Caro, A. Niño, Biophys. Chem. 2006, 124, 155-160. 